\title{
Uma Revisão Sistemática das Tecnologias da Web Semântica em Ambientes Educacionais
}

\author{
Aparecida M. Zem-Lopes, Lais Z. Pedro, Fernando R. H. Andrade, \\ Simone S. Borges, Seiji Isotani \\ Instituto de Ciências Matemáticas e de Computação - Universidade de São Paulo (USP) \\ Avenida Trabalhador São-Carlense, 400 - São Carlos - SP - Brazil \\ \{cidazem, laisp, fernando.heb, sborges, sisotani\}@icmc.usp.br
}

Resumo. As pesquisas sobre ambientes educacionais baseados na Web têm avançado nos últimos anos, impulsionadas pela evolução das Tecnologias de Informação $e$ Comunicação. Além disso, a Web Semântica, que faz uso de ontologias e taxonomias para representar conhecimento e anotar as informações contidas na Web e permite que as informações conectadas passem a ter significado compreensível e compartilhável, por pessoas e computadores, tem despertado o interesse dos pesquisadores. Contudo, poucos trabalhos discutem, apresentam e catalogam os resultados já obtidos na comunidade. Assim, o objetivo deste trabalho foi conduzir uma Revisão Sistemática com o propósito de identificar onde e como tecnologias da Web Semântica vêm sendo utilizadas em ambientes educacionais. A busca por artigos foi realizada nas bases eletrônicas mais relevantes na área de Computação e Educação. Inicialmente, 445 artigos foram selecionados. Após a leitura e aplicação dos critérios de inclusão/exclusão restaram 92 artigos. Os resultados das análises realizadas a partir destes trabalhos fornecem um panorama sobre a aplicação da Web Semântica em sistemas educacionais e destacam a importância do desenvolvimento elou uso de ontologias nesse tipo de sistema. Entretanto, verificou-se que poucas ontologias estão devidamente documentadas elou acessíveis, o que pode inviabilizar seu reuso.

Abstract. Research on Web-based educational environments has advanced in recent years, driven by the evolution of Information Technologies and Communication. Semantic Web uses ontologies and taxonomies to represent knowledge and write down the specifications of the Web This approach allows information to be shared, and provide meaning understandable by people and computers. For these reasons, the Semantic Web have attracted the interest of researchers.However, few articles present and catalog the results already obtained in the community. This study aims to conduct a systematic review in order to identify where and how Semantic Web technologies are being used in educational settings. The search for articles was conducted in the most relevant electronic databases in Computer Science and Education. Initially, 445 articles were selected. After reading and applying the criteria for inclusion/exclusion remaining 92 articles. The results of the 
analyzes from these articles provide an overview on the application of Semantic Web in educational systems and highlight the importance of developing and/or use of ontologies in this type of system. However, it was found that few ontologies are properly documented and/or accessible, which may hinders their reuse.

\section{Introdução}

Os estudos de ambientes educacionais baseados na web têm avançado nos últimos anos, impulsionados pela evolução das Tecnologias de Informação e Comunicação, despertando interesse da comunidade com relação a temas ligados à Educação a Distância, Informática na Educação, entre outros (Bittencourt et al., 2008). O trabalho de Abreu et al. (2012) evidencia esse aspecto ao realizar um Revisão Sistemática para investigar quais tecnologias vêm sendo utilizadas como suporte no desenvolvimento de softwares educativos. Lima et al. (2012) também levantaram, por meio de uma revisão sistemática, quais técnicas e processos têm sido utilizados para desenvolver softwares educativos.

A crescente evolução das pesquisas em Web Semântica tem apresentado um grande potencial para resolver diversos problemas nos ambientes de ensino e aprendizagem baseados na web atual como, por exemplo, a falta de interoperabilidade entre sistemas e as dificuldades para criar, compartilhar e reutilizar conteúdos e informações disponíveis na rede. Desse modo, a web que conecta apenas informações por intermédio de links entre páginas, tende a se transformar na web que conecta "conhecimento", por meio do uso de técnicas da Web Semântica (Devedzic, 2006). Assim, o uso de ontologias e taxonomias para representar conhecimento e anotar a informação contida na web permite que essas informações conectadas passem a ter significados que são compreensíveis e compartilháveis por pessoas e computadores.

Essa capacidade possibilita o desenvolvimento de novos mecanismos de aprendizagem, inferências e análise sobre o nível de conhecimento do aluno, além de viabilizar o uso de agentes inteligentes para lidar com os conteúdos educacionais contidos na web de forma mais rápida e efetiva (Isotani et al., 2009). Além disso, as ontologias permitem a representação semântica dos dados e tornam possível a elaboração de uma rede de conhecimento humano que pode ser processado e consumido, também, por agentes computacionais. Porém, ainda existe uma grande dificuldade em desenvolver sistemas educacionais integrados que permitam o compartilhamento e o reaproveitamento de conteúdos.

Em face disso, conhecer o estado da arte e o atual patamar da utilização da Web Semântica em aplicações educacionais apresenta-se como uma necessidade para construir uma base sólida sobre essa área de pesquisa.

Assim, essa Revisão Sistemática (RSL) tem por objetivo identificar, a partir dos estudos primários coletados, onde e de que forma as tecnologias da Web Semântica vem sendo utilizadas em ambientes educacionais. 


\section{Metodologia}

Para conduzir essa RSL, seguiu-se o processo proposto por Kitchenham (2004). Esse processo apresenta três fases principais: Planejamento, Condução e Relatório.

$\mathrm{Na}$ fase de planejamento, a partir do estabelecimento do objetivo da pesquisa, deuse início ao desenvolvimento do protocolo, utilizado para orientar a coleta de evidências da RSL. Devido a questões de limitação do número de páginas, serão apresentadas apenas as seções mais importantes desse protocolo ${ }^{1}$. O protocolo completo faz parte do relatório técnico e contém os demais documentos utilizados na Revisão Sistemática, tais como: a lista artigos incluídos nas duas fases de seleção, os artigos rejeitados e a relação dos artigos que compõe o grupo de controle.

\subsection{Questões de pesquisa}

As questões de pesquisa foram elaboradas a partir do objetivo da RSL, junto a especialistas da área, os quais também são responsáveis por estabelecer um "grupo de controle", ou seja, um conjunto de trabalhos relevantes para o tema da revisão que deveria, necessariamente, ser retornado pelas máquinas de busca. Desse modo, foram elaboradas a questão de pesquisa principal e outras cinco questões secundárias:

QP $\left.\mathbf{P}_{1}\right)$ Existem ambientes educacionais baseados em Web Semântica?

A partir da questão principal, foram elaboradas as questões secundárias:

QP $\left.\mathbf{P}_{1.1}\right)$ Há algum sistema tutor inteligente baseado em Web Semântica desenvolvido?

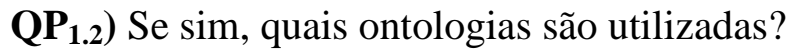

$\left.\mathbf{Q P}_{1.3}\right)$ Há algum guideline ou framework que contribui para implementação de ambientes educacionais baseados em Web Semântica desenvolvido?

QP $\left.\mathbf{P}_{1.4}\right)$ Quais as principais abordagens, metodologias e técnicas utilizadas para implementar os ambientes virtuais de ensino e aprendizagem, baseados nesta tecnologia?

\subsection{Estratégias e processo de busca}

A string de busca foi construída de acordo com Kitchenham (2006), por meio da identificação das principais palavras-chave derivadas das questões de pesquisa. Utiliza-se os operadores OR e AND, respectivamente para termos sinônimos e termos alternativos de cada palavra chave, definidos como "Semantic Web" and "educational environment". Para validar os termos, foi solicitada a ajuda de um pesquisador especialista na área em questão. Obteve-se, então, como resultado, a seguinte string de busca genérica:

(("semantic web" or "web semantics" or "semantic wiki" or "semantic computing") and ("educational environment" or "distance education" or "educational system" or "educational systems" or "intelligent tutoring system" or "e-learning environment" or "mlearning environment" or "elearning environment" or "mlearning environment" or "virtual

\footnotetext{
${ }^{1}$ Protocolo disponível no endereço: <https://dl.dropbox.com/s/7acfjg5fvqj6iie/workshop_2013.html>
} 
learning environment" or "teaching and learning" or "learning and teaching" or "adaptive learning" or "collaborative learning" or "intelligent virtual environment")))

A escolha das bases eletrônicas para as buscas foi baseada em Dieste et al. (2009), que estabelece alguns critérios como a disponibilidade dos estudos primários, cobertura das publicações e conferências relevantes na área de pesquisa, e a busca por estudos no idioma inglês (adotada nos principais eventos e periódicos científicos) e em português, para tentar recuperar algum estudo importante no Brasil. As máquinas de busca digitais utilizadas, consideradas relevantes para a área, foram: ACM Digital Library, IEEE Xplore, Elsevier (via ScienceDirect), Springer (via SciVerse) e Scopus, todas susceptíveis a conter importantes estudos primários.

A string genérica foi devidamente ajustada para cada base eletrônica selecionada, de acordo com as características e especificidades das mesmas. Ainda, de acordo com os artigos estabelecidos no grupo de controle, foi possível validar se as bases eletrônicas selecionadas estavam de acordo com o objetivo da RSL.

\subsection{Processo de seleção dos trabalhos}

Foram selecionados estudos que abordam e documentam aspectos práticos da utilização da Web Semântica, tais como: propostas de ontologias para educação, metodologias de implementação, estudos experimentais, guidelines e frameworks. Estudos que apenas abordem aspectos teóricos, filosóficos ou pedagógicos da utilização de Web Semântica para desenvolvimento de ambientes educacionais serão excluídos.

A seleção e extração de dados foram conduzidas por quatro pesquisadores e as diferenças e dúvidas solucionadas por consenso. Desse modo, a seleção inicial dos estudos deu-se mediante a leitura do título, resumo e palavras chave dos estudos e a aplicação dos critérios de inclusão e exclusão, apresentados na Seção 2.4. Depois, por meio da leitura completa dos estudos, houve a reaplicação dos critérios de inclusão e exclusão para a extração dos dados. Para isso, os estudos encontrados em duplicidade foram prontamente eliminados, mantendo como registro apenas a referência às bases em que foram encontrados.

$\mathrm{Na}$ a extração dos dados, foi utilizado um formulário elaborado na ferramenta GoogleDocs, e, durante uma reunião para esclarecimento de diferenças, os mesmos foram reavaliados e inseridos na ferramenta $\mathrm{START}^{2}$.

O processo da RSL pode ser resumido em:

1. Seleção inicial - Leitura do título, resumo e palavras-chave

2. Aplicação dos critérios de Inclusão e Exclusão

3. 2a. Fase da Seleção

- Leitura completa dos estudos

4. Reaplicação dos critérios de Inclusão e Exclusão

${ }^{2}$ StArt - disponível em <http://lapes.dc.ufscar.br/tools/start_tool> 
5. Extração dos Dados

- Análise de qualidade

6. Análise dos Dados

\subsection{Critérios de inclusão e exclusão}

De acordo com o protocolo definido para essa RSL, foram estabelecidos os critérios de inclusão e exclusão para garantir a seleção de estudos relevantes que pudessem responder às questões de pesquisa.

\section{Critérios de Inclusão:}

IC1 - Documentos que abordem e documentem aspectos práticos da utilização de Web Semântica, tais como: propostas de ontologias para educação, metodologias de implementação, estudos de caso sobre Web Semântica em educação, guidelines para Web Semântica em educação e frameworks para Web Semântica em educação;

IC2 - O artigo apresenta um guideline para a utilização de Web Semântica em ambientes educacionais;

IC3 - O artigo apresenta um sistema tutor inteligente baseado em Web Semântica;

IC4 - O artigo apresenta um framework para a utilização de Web Semântica em ambientes educacionais;

IC5 - O artigo apresenta uma ontologia para ambientes de ensino;

IC6 - O artigo apresenta desafios encontrados em um ambiente de ensino baseado em Web Semântica; e

IC7 - O artigo apresenta o desenvolvimento de um ambiente de ensino semântico.

\section{Critérios de Exclusão:}

EC1 - Estudos que não estejam na língua portuguesa ou inglesa;

EC2 - Relatórios técnicos, documentos que estão disponíveis na forma de resumos ou apresentações e também revisões de literatura secundárias (ou seja, revisões e mapeamentos sistemáticos da literatura);

EC3 - Estudos que não possuírem versões completas disponíveis;

EC4 - Estudos que abordam apenas aspectos teóricos ou filosóficos sobre a utilização de Web Semântica para desenvolvimento de ambientes educacionais; e

EC5 - Estudos que não apresentam relação com sistemas educacionais baseados em Web Semântica.

\section{Resultados e análise dos dados}

Essa seção apresenta os resultados encontrados na Revisão Sistemática realizado, a partir dos estudos primários recuperados, e uma breve análise e discussão desses estudos. 


\subsection{Informações gerais sobre os estudos primários}

A string foi devidamente adaptada, de acordo com as especificidades de cada uma das bases eletrônicas selecionadas, calibrada e, a partir daí, as buscas foram realizadas e totalizaram a recuperação de 445 estudos.

$\mathrm{O}$ resultado das pesquisas foi exportado em cada base eletrônica e importado na ferramenta START. Essa distribuição inicial dos estudos por base, em quantidade e percentual, pode ser observada na Figura 1. A ferramenta START realizou, automaticamente, a identificação dos estudos duplicados e disponibilizou apenas uma das cópias para aplicação dos critérios de inclusão e exclusão. Nessa primeira etapa foram eliminados 47 artigos por duplicidade.

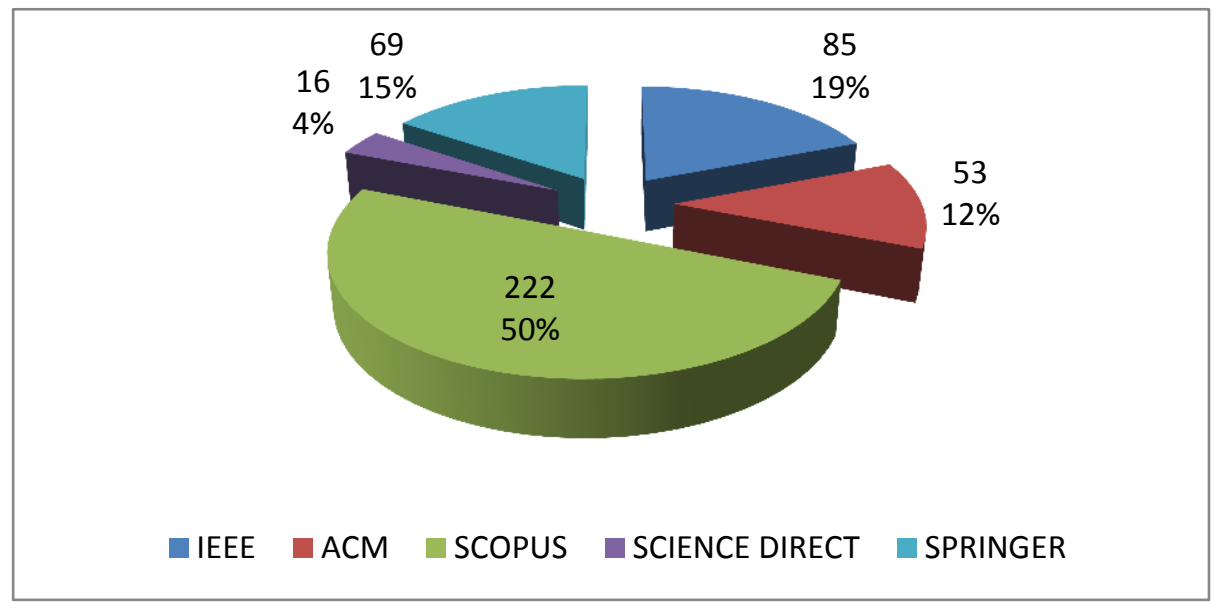

Figura 1: Distribuição percentual dos estudos recuperados por base eletrônica

Ao final da primeira etapa de seleção, dos 445 estudos recuperados em todas as fontes, 173 estudos foram incluídos. Após essa etapa, procedeu-se a segunda seleção dos estudos, em que foi realizada a leitura completa por cada pesquisador e, por meio de um formulário de extração, foram obtidas as informações relevantes para responder às questões de pesquisa do trabalho. Dessa forma, obteve-se um total de 92 estudos incluídos para análise.

A Tabela 1 mostra, de forma resumida, a evolução do processo de seleção dos estudos primários. Na primeira coluna estão listadas as máquinas de busca que foram utilizadas neste estudo e, na segunda, a quantidade de artigos recuperados na primeira etapa do processo. A terceira e quarta colunas representam, respectivamente, a quantidade de artigos incluídos, excluídos e duplicados na primeira seleção, com a ajuda da ferramenta START. Finalmente, as duas últimas colunas mostram o número de estudos incluídos e excluídos, na segunda etapa da seleção, a partir da leitura completa.

Tabela 1 - Resumo do processo de seleção dos estudos primários 


\begin{tabular}{|l|cc|c|c|c|c|c|}
\hline \multirow{2}{*}{ Base } & \multirow{2}{*}{ Inicial } & \multicolumn{3}{c|}{$\mathbf{1}^{\mathbf{a}}$ seleção } & \multicolumn{2}{c|}{$\mathbf{2}^{\mathbf{a}}$ seleção } \\
\cline { 3 - 8 } & & incluidos & excluidos & duplicados & incluidos & excluidos \\
\hline IEEE & 85 & $(19 \%)$ & 31 & 45 & 9 & 20 & 11 \\
\hline ACM & 53 & $(22 \%)$ & 25 & 25 & 3 & 13 & 12 \\
\hline SCOPUS & $222(50 \%)$ & 97 & 103 & 22 & 47 & 50 \\
\hline ELSEVIER & $16(4 \%)$ & 8 & 0 & 8 & 6 & 2 \\
\hline SPRINGER & $69(16 \%)$ & 12 & 52 & 5 & 7 & 5 \\
\hline
\end{tabular}

Além dos critérios de inclusão e exclusão estabelecidos, foi realizada a avaliação de qualidade dos estudos, que permitiu classificá-los de acordo com sua relevância com relação ao objetivo da RSL, completude, documentação e aspectos práticos abordados por eles. As notas foram atribuídas como a soma dos pesos fornecidos para os critérios de inclusão atribuídos aos artigos. A descrição completa e demonstração das tabelas de relações de notas, está disponível no relatório técnico ${ }^{3}$ dessa Revisão Sistemática da Literatura.

\subsection{Respostas às questões de pesquisa}

Esta seção apresenta as evidências encontradas nos estudos primários que contribuem para responder às questões propostas nessa RSL.

\section{QP 1 : Há algum ambiente educacional baseado em Web Semântica?}

Após a extração e sumarização dos dados, foi possível constatar que os 92 estudos estão relacionados à Web Semântica e/ou educação. Esses estudos foram, então, classificados de acordo com a Figura 2.

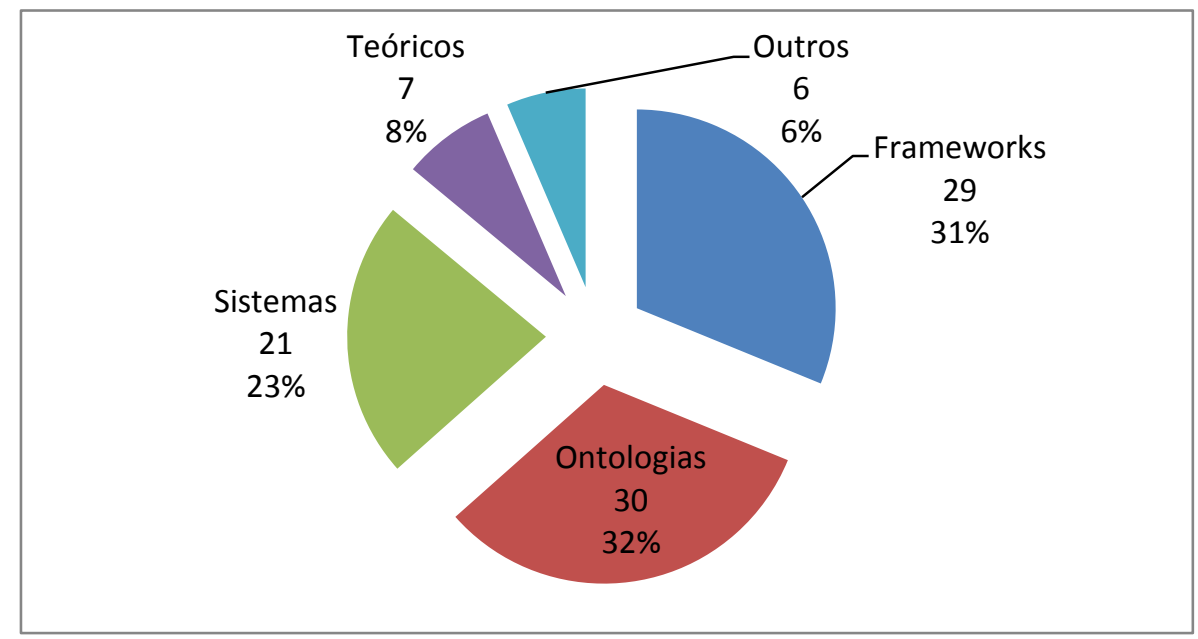

Figura 2 - Tipos de estudos para extração dos dados

Assim, concluiu-se que existem diversos esforços dedicados à criação e testes de

\footnotetext{
${ }^{3}$ Relatório técnico: 〈http://goo.gl/jMbS1m〉
} 
sistemas educacionais (23\%), frameworks $(31 \%)$ e ontologias $(32 \%)$, além de outros não nomeados, que utilizam a Web Semântica para facilitar o reuso de conhecimento e o aprimoramento de agentes inteligentes, o que permite inferir sobre as questões secundárias desse trabalho. Desses 93 estudos, 40\% tratam ou apresentam mais que uma abordagem (e.g. framework e ontologia ou sistema e framework ou, ainda, framework e sistema).

QP 1.1) Há algum sistema tutor inteligente baseado em Web Semântica desenvolvido?

Por meio da questão do formulário respondido "Qual tipo de ambiente foi tratado no artigo", foi possível observar que já existem sistemas tutores inteligentes semânticos em desenvolvimento, além de expectativas sobre os benefícios que a Web Semântica pode proporcionar para a melhoria da performance dos agentes inteligentes. Dentre os artigos analisados, 12 deles (13\%) tratam Sistemas Tutores Inteligentes (STI) baseados em Web Semântica, já que apresentam alguma forma de personalização de aprendizagem (na planilha dos estudos selecionados $2^{a}$ etapa, é possível identificar uma aba com os estudos que tratam dos STI dessa RSL) ${ }^{4}$. Um dos trabalhos é apresentado por Rosié et. al (2004), que propõem um sistema tutor inteligente baseado na simulação de professores reais no processo de ensino e aprendizagem, no qual implementam os conceitos de interoperabilidade do domínio do conhecimento.

\section{QP $\left.\mathbf{P}_{1.2}\right)$ Quais ontologias são utilizadas?}

A utilização de ontologias apropriadas para sistemas educacionais apresentou-se como uma importante preocupação nos estudos recuperados, já que 46 deles $(50 \%)^{5}$ fazem menção ao uso de ontologias especificas para ambientes educacionais. Foi possível identificar, nos estudos, as ontologias Fedora, Mulgara, G.O. (Gene Ontology), SCORM, SKOS, LOCO, LOCO-cite, UMMO, GUMO, SIOC, ALoCOM, COES, AVATAR entre outras. Os pesquisadores Mitrovic and Devedzic (2002) propuseram um modelo de ontologia denominado M-OBLIGE, capaz de prover uma infraestrutura que possibilite o reuso e compartilhamento de conhecimento em sistemas tutores inteligentes, afirmando, ainda, que esse modelo é o primeiro passo para o desenvolvimento de um framework unificado que habilite a interoperabilidade entre múltiplos sistemas tutores na web.

QP $\left.\mathbf{P}_{1.3}\right)$ Há algum guideline, framework que contribuem para implementação de ambientes educacionais baseados em Web Semântica desenvolvido?

Segundo os dados coletados, não existem propostas de guidelines. Entretanto, foram encontradas diversas iniciativas propondo frameworks para ambientes educacionais utilizando Web Semântica, totalizando 40 artigos (43\% dos estudos) ${ }^{6}$. Cubric et al. (2009), por exemplo, apresentam um framework semântico que pode ser utilizado para gerar ambientes web, e-learning e e-assessment para qualquer domínio de conhecimento. Já Conesa et al. (2012) trazem o framework ALICE, baseado em ontologias, que inclui informações emocionais sobre o sentimento e opiniões dos estudantes enquanto ocorre a

\footnotetext{
${ }^{456}$ Estudos aceitos $2^{\mathrm{a}}$ etapa: 〈http://goo.gl/RU0XrB >
} 
colaboração no sistema.

QP $\left.\mathbf{P}_{1.4}\right)$ Quais as principais abordagens, metodologias e técnicas utilizadas para implementar os ambientes de virtuais de ensino e aprendizagem?

Os métodos para a implementação de ambientes educacionais não estão bem definidos e apresentam uma grande variação, de acordo com os objetivos do sistema proposto. Isso pode ser verificado pela falta de guidelines e pela diversidade de frameworks distintos e também pelas diferenças existentes entre os domínios de aplicação dos ambientes de ensino encontrados. Dentre os estudos avaliados, os principais tópicos que têm atraído a atenção da comunidade são ligados à melhoria de agentes inteligentes para a recomendação de conteúdo apropriados aos alunos, à melhoria dos módulos pedagógicos de sistemas adaptativos e ao reuso e compartilhamento de conteúdos entre modelos de domínios de sistemas distintos (Mitrovic and Devedzic, 2002; Dung and Florea, 2011; Strujic and Sendelj, 2012).

\section{Considerações finais}

Os dados extraídos forneceram evidências importantes sobre o panorama de aplicação da Web Semântica em sistemas educacionais e demonstram que, por estar sendo explorada por apenas algumas iniciativas, sua utilização está pouco consolidada. Os resultados mostraram que diversas ontologias estão sendo desenvolvidas para modelar os objetos de aprendizagem, os perfis dos alunos e as práticas pedagógicas. Entretanto, poucas dessas ontologias continham informações disponíveis sobre acesso e localização (apenas cinco, das trinta e sete ontologias identificadas nos dados extraídos), de modo que não atendem às características de reuso e compartilhamento. Outra observação feita durante a condução da revisão é a diversidade de frameworks propostos para incorporar a Web Semântica aos ambientes educacionais, o que confirma a preocupação e interesse da comunidade científica em oferecer ferramentas que possam contribuir para otimizar a qualidade desses ambientes.

\section{Referências Bibliográficas}

Abreu, F.; Almeida, A.; Barreiros, E.; Saraiva, J.; Soares, S.; Araújo, A.; Henrique, G. (2012) "Métodos, Técnicas e Ferramentas para o Desenvolvimento de Software Educacional: Um Mapeamento Sistemático". Anais do $23^{\circ}$ Simpósio Brasileiro de Informática na Educação (SBIE 2012), ISSN 2316-6533, Rio de Janeiro, 26-30 de Novembro de 2012.

Bittencourt, I. I., Isotani, S., Costa, E. \& Mizoguchi, R. (2008). "Research Directions on Semantic Web and Education”. Journal Scientia, 19(1), 59-66.

Conesa, J; Caballé, S; Gañan, D; Prieto, J. (2012) "Towards the Representation of Emotional Information from On-line Collaborative Learning Sessions". Sixth International Conference on Complex, Intelligent, and Software Intensive Systems. Barcelona, Spain.

Cubric, M; Tripathi, V. (2009) “A Semantic Web Framework for Generating Collaborative E-learning Environments". International Journal of Emerging Technologies in Learning, v. 4 (3), p. 4. 
Devedzic, V. “Semantic Web and Education”. Springer, 2006.

Dieste, O.; Grimán, A.; Juristo, N. (2009). "Developing search strategies for detecting relevant experiments". Empirical Software Engineering, v. 14, n. 5, p. 513-539, 2009.

Dung, P. Q.; Florea, A. M. (2011) “An architecture and a domain ontology for personalized multi-agent e-learning systems", Third International Conference on Knowledge and Systems Engineering, IEEE Computer Society, DOI 10.1109/KSE.2011.35.

Isotani, S.; Bittencourt, I. I.; Mizoguchi, R.; Costa, E. (2009). "Estado da Arte em Web Semântica e Web 2.0: Potencialidades e Tendências da Nova Geração de Ambientes de Ensino na Internet”. Revista Brasileira de Informação na Educação, 17, p. 30-42.

Kitchenham, B. (2004). "Procedures for performing systematic reviews". Technical Report TR/SE- 0401 and NICTA Technical Report 0400011T.1, Keele University and National ICT Australia Ltd.

Kitchenham, B. (2006). "Empirical paradigm - the role of experiments". Empirical software engineering issues: critical assessment and future directions, Heidelberg. SpringerVerlag.

Kitchenham, B. ; Charters, S. (2007). "Guidelines for performing systematic literature reviews in software engineering". Technical Report EBSE 2007-001, Keele University and Durham University Joint Report. http://www.dur.ac.uk/ebse/resources/Systematicreviews-5-8.pdf.

Lima, M. M. de; Lima, A. R. d; Monteiro, A. C. C.; Cavalcante Jr, E. H.; Gomes, L. de Q. L. (2012) "Uma Revisão Sistemática da Literatura dos Processos de Desenvolvimento de Software Educativo". Anais do $23^{\circ}$ Simpósio Brasileiro de Informática na Educação (SBIE 2012), ISSN 2316-6533, Rio de Janeiro, 26-30 de Novembro de 2012.

Mitrovic, A.; Devedzic, V. (2002) “A Model of Multitutor Ontology-Based Learning Environments". Proceedings of the International Conference on Computers in Education (ICCE'02) 0-7695-1509-6/02. IEEE, 2002.

Petersen, K.; Feldt, R.; Mujtaba, S; Mattsson, M. "Systematic Mapping Studies in Software Engineering”. EASE 2008. Anais da $12^{\mathrm{a}}$ Conferência Internacional sobre Avaliação e Avaliação em Engenharia de Software, 68-77.

Rosié, M; Glavinic, V; Stankov, S. (2004) "Intelligent Tutoring Interoperability for the New Web”. IEEE Melecon 2004. Dubrovnik. Croatia, 2004.

Strujic, D.; Sendelj, R. (2012) "Concepts of modern systems for e-learning based on sematic technologies", Mediterranean Conference on Embedded Computing MECO 2012, Bar, Montenegro.

Travassos, G. H.; Gurov, D.; Amaral, E. A. G. (2002). "Introdução à engenharia de software experimental". Relatório técnico RT-ES-590/02. Programa de engenharia de sistemas e computação. COOPE. UFRJ. Rio de Janeiro, 2002. 